\title{
EASD
}

\section{EUROPEAN ASSOCIATION FOR THE STUDY OF DIABETES}

ASSOCIATION EUROPEENE POUR L'ETUDE DU DIABETE · EUROPÄISCHE GESELLSCHAFT FÜR DIABETOLOGIE

Rheindorfer Weg 3 - D-40591 Düsseldorf · Germany · Tel: +49-211-7 584690 - Fax: +49-211-75 846929

E-mail: easd@uni-duesseldorf.de·Homepage: http://www.easd.org

\section{Announcements}

\section{4th International Symposium on Diabetes Mellitus and its Complications: From Molecular Biology to Clinical Medicine}

University of Central Lancashire, Preston, UK, 7-8 July 2003

This symposium is organised by the University of Central Lancashire and Lancashire Teaching (NHS) Trust in collaboration with Diabetes and Cardiovascular Research Group, FMHS, UAE University, Al Ain, UAE, Al Ain Diabetes Research Group, Al Ain, UAE.

\section{Abstracts:}

Please send your abstracts by e-mail or on disc by 15 April 2003 to either:

Liz Kelly

Conference Officer

University of Central Lancashire

Preston PR1 2HE

UK

Tel: +44-1772-892656

Fax: +44-1772-892977

E-mail: ejkelly@uclan.ac.uk

or

Emma Woodward

Conference Officer

University of Central Lancashire

Preston PR1 2HE

UK

Tel: +44-1772-892654

Fax: +44-1772-892977

E-mail: eawoodward@uclan.ac.uk

\section{An International Symposium: Digestive Hormones, Appetite and Energy Balance}

Imperial College London, 30 June-1 July 2003

Deadline for abstract submission: 30 April 2003

\section{Location:}

Wolfson Conference Centre

Imperial College London

Hammersmith Hospital Campus

Du Cane Road

London W12 0NN

UK
For more information, please contact:

Tel: +44-20-83833242

E-mail: r.boning@imperial.ac.uk

http://www.obesity.med.imperial.ac.uk

\section{9th Bart's International Symposium}

"Prevention of Type $\mathbf{2}$ diabetes and its progression"

Robin Brook Centre, St Bartholomew's Hospital,

London, UK, 11 April 2003, 9:00-17:00

This year's symposium is chaired by RDG Leslie and $P$ Pozzilli and aims to present emerging strategies for therapy and prevention of diabetes.

For further information, please contact:

Symposium Organiser

Department of Diabetes and Metabolism

$3^{\text {rd }}$ Floor

Dominion House

59 Bartholomew Close

West Smithfield

London EC1A 7BE

UK

Tel: +44-171-6017450

Fax: +44-171-6017449

E-mail: j.e.gough@mds.qmw.ac.uk

\section{$1^{\text {th }}$ International Symposium on the Autonomic Nervous System}

St. Thomas, U.S. Virgin Islands, 5-8 November, 2003

For further information, please contact:

Anita Zeller

AAS Executive Secretary

American Autonomic Society

$5458193^{\text {rd }}$ Street

W. Farmington, MN 55024

USA

E-mail: zeller@mayo.edu

Homepage: www.americanautonomicsociety.org 